\title{
ESHA et le Livre Blanc sur les énergies renouvelables
}

\author{
ESHA and the White Paper on renewable energies \\ par Henri Baguenier \\ Président l'Association Européenne pour la Petite Hydraulique (ESHA)
}

L'ESHA (European Small Hydropower Association) estimates that some topics should have been developed in the White Paper on renewable energies written for the European Commission:

- the necessity of applying the proposed measures to reach the objectives because the renewable energies are not competitive at the present time,

- objectives for each country,

- a key action for the small hydropower.

Après avoir pris connaissance du Livre Blanc sur les énergies renouvelables de la Commission européenne, ESHA estime que le document pourrait être enrichi s'il était tenu compte des éléments détaillés ci-dessous.

\section{I $\square$ PERTE DE COMPÉTITIVITÉ DES ÉNERGIES RENOUVELABLES}

Le Livre Blanc ne souligne pas assez la perte de compétitivité, ces dernières années, du moins pour la production d'électricité, des énergies renouvelables, par rapport à la production d'électricité d'origine fossile. En effet, en dépit des baisses des coûts de production de l'électricité générée par les énergies renouvelables, les baisses de coûts beaucoup plus importantes de production de l'électricité d'origine fossile ont renforcé la compétitivité de celle-ci. Ce gain de compétitivité est le résultat conjugué de la baisse des prix des énergies fossiles et de la baisse des coûts techniques de génération (grâce en particulier aux énormes progrès du cycle-combiné, mais aussi de la non prise en compte dans les coûts de production des externalités négatives des énergies fossiles (la Commission n'ayant pu imposer ses vues sur la taxe énergie/ $\mathrm{CO}_{2}$ ). A cette perte de compétitivité des énergies renouvelables s’ajoutent les conséquences de la déréglementation du secteur électrique, qui dans de nombreux pays entraîne la remise en cause des obligations et conditions d'achat de l'électricité générée par les énergies renouvelables (les prix de rachat de l'électricité d'origine renouvelable se sont en général fortement dégradés ces dernières années).

Le Livre blanc devrait donc affirmer beaucoup plus frontalement que, sans la mise en cuvre des mesures qu'il avance, les objectifs proposés ne seront pas atteints. En particulier, nous pensons aux mesures concernant les garanties de prise par les réseaux de l'électricité à un prix convenable reflétant pour le moins les coûts évités de long terme (ou " City Gate ") et les coûts externes associés à la génération d'électricité d'origine fossile. La nécessité d'une Directive en la matière doit être encore plus affirmée et sa préparation initiée dans les plus brefs délais.

\section{II — NÉCESSITÉ DE FIXER DES OBJECTIFS PAR PAYS}

Le Livre blanc fixe des objectifs par technologie pour I'UE, mais ne décompose pas ces objectifs par pays. Nous comprenons qu'à ce stade, la commission n'ait pas voulu définir les objectifs de chaque pays, mais elle devrait inviter, en usant de son pouvoir de proposition, chacun des membres de l'Union européenne à présenter dans un délai raisonnable (p.ex. un an) sa contribution à chacun de ces objectifs. Sans un engagement du Conseil dans ce sens (qui serait cohérent avec les engagements par pays sur les quotas d'émissions de gaz à effet de serre), les objectifs risquent de n'engager que les auteurs du Livre blanc.

\section{I LA PETITE HYDRAULIQUE, UN PEU OUBLIÉE}

Comme citoyen convaincu que les énergies renouvelables, tout comme l'efficacité énergétique, sont une composante majeure de toute stratégie de long terme d'une politique énergétique visant à la sécurité des approvisionnements et à la protection de l'environnement, je ne peux que me réjouir de la volonté affirmée de la Commission de promouvoir globalement les énergies renouvelables. Comme Président de l'Association Européenne de Petite Hydraulique (petites centrales hydroélectriques de moins de $10 \mathrm{MW}$ ), je suis encore une fois désolé de constater que la petite hydraulique (son 
développement) continue à ne pas être une priorité pour la Commission. Cette moindre importance apportée aux petites centrales hydroélectriques (je souligne cependant qu'il y a eu un progrès par rapport au Livre vert sur les énergies renouvelables et encore plus par rapport au Livre blanc sur la politique énergétique qui n'en parlait pas du tout) se reflète notamment dans l'absence de proposition concernant la petite hydraulique au niveau des " key actions " proposées pour le photovoltaïque, les fermes éoliennes, la biomasse et les " cités solaires". Il me semble donc encore une fois nécessaire de souligner les raisons pour lesquelles le développement de la petite hydraulique à l'intérieur et à l'extérieur de l'Union européenne doit être une priorité dans le cadre des objectifs définis pour les énergies renouvelables.

- La petite hydraulique est aujourd'hui au premier rang de la génération d'électricité d’origine renouvelable (évidemment hors grande hydraulique) dans l'Union européenne et dans le monde. Ainsi la puissance installée dans l'Union européenne est actuellement de $\pm 10000 \mathrm{MW}$ (-de 3500 pour les éoliennes) et de \pm 45000 au niveau mondial (dont 21000 pour la seule RP de Chine). Les perspectives de développement proposées pour le Livre blanc pour 2010 sont de $5000 \mathrm{MW}$ supplémentaires dans l'Union européenne et le potentiel à développer au niveau mondial dépasse les $100000 \mathrm{MW}$. Il est donc clair que la croissance globale de l'électricité d'origine renouvelable passe également par la croissance de celle qui restera à l'horizon 2010 de loin la première renouvelable pour la production d'électricité au niveau mondial.

- Les entreprises de l'Union européenne, qu'il s'agisse des fabricants d'équipement, des bureaux d'ingénieurs ou des investisseurs, sont encore leader au niveau des petites centrales hydrauliques et donc bien placées pour conquérir des parts de marché dans les pays tiers où se situe l'essentiel du potentiel à développer. L'histoire industrielle nous enseigne qu'une condition essentielle de la conquête de marché extérieur passe par le maintien d'un solide marché intérieur. Dans le domaine de la petite hydraulique, il est clair pour nous que le soutien du marché intérieur est une condition fondamentale du maintien du leadership sur l'extérieur. Comme nous le notions dans le premier commentaire de notre lettre, les conditions économiques faites aux petites centrales hydrauliques se sont globalement (avec quelques exceptions pour certains pays) dégradées ces dernières années et des obstacles croissants (essentiellement contraintes administratives) bloquent, dans de nombreux pays, le développement de nouveaux sites (il convient de souligner que ces mêmes problèmes affectent le développement de l'énergie éolienne). Ainsi, confrontés à une rentabilité moindre et des obstacles croissants pour la réalisation de projets, les investisseurs le plus souvent renoncent et nous ne pouvons que réaffirmer que dans un scénario " doing nothing "les objectifs proposés par le Livre blanc pour les petites centrales hydrauliques ne seront pas atteints (comme ceux pour 2005 du programme ALTENER ne l'auront pas été). Le rétrécissement progressif de ce marché intérieur a, comme corollaire, la diminution d'activité des entreprises, comme l'atteste la fermeture de différentes fabriques d'équipements (principalement turbiniers). L'Union européenne, au-delà de la non-réalisation de ses objectifs intérieurs, risque également de perdre les marchés prometteurs tels que les marchés chinois, brésiliens, indiens, qui rappelons-le se chiffrent en milliers de MW.

- Les petites centrales hydrauliques sont pourtant considérées (voir les études sur les externalités des filières énergétiques promues par le Parlement européen) parmi les moins dommageables pour l'environnement par $\mathrm{kWh}$ produit, leur technologie est mature, les coûts sont bien contrôlés, elles représentent dans les 15 prochaines années le principal marché à l'exportation pour les énergies renouvelables. Elles devraient donc être dans les priorités définies par le Livre blanc.

Pour toutes ces raisons, nous pensons que, en accord d'ailleurs avec la philosophie du Livre blanc, la version finale de celui-ci devrait intégrer une "key-action" au niveau de la promotion de la petite hydraulique dans les pays tiers. Cette "key-action" pourrait comprendre des soutiens à l'identification de potentiels, à la création des cadres légaux et institutionnels favorables, des crédits d'investissement, des soutiens aux études de faisabilité, etc. Parallèlement à cette " key action ", la directive concernant les rapports entre réseaux électriques et production à partir d'énergies renouvelables devrait être accompagnée, comme le proposait déjà l'ESHA en 1995, d'une directive concernant les conditions administratives de développement de projets d'énergie renouvelable dans l'Union européenne.

En dehors de ces mesures, nous pensons plus simplement que la petite hydraulique doit être pleinement intégrée dans tous les programmes de l'Union européenne ayant trait au développement des énergies renouvelables à l'intérieur et à l'extérieur de l'Union européenne. 